\title{
Peer-to-peer systems for video delivery
}

\author{
Maria Luisa Merani
}

Received: 9 June 2010 / Accepted: 16 June 2010 / Published online: 6 July 2010

(C) Springer Science+Business Media, LLC 2010

In the very recent years, peer-to-peer for streaming video applications has gained considerable attention: commercial systems have appeared, raising interest among a community of potential users that have quickly increased and reached an impressive size. The pioneering experience of the Coolstreaming prototype has been soon paired by popular systems such as PPlive, Sopcast and PPStream, platforms capable of attracting thousands of users to watch video programs over the Internet.

Among academia too, peer-to-peer streaming architectures have spurred curiosity: a significant body of papers has been produced, bringing forth studies on heterogeneous aspects related to these systems.

Nevertheless, much work remains to be done. The field of modeling is only partly explored; the understanding of the basilar laws that shape the behaviour of these systems is far from being satisfying; the mutual relation between system performance and the different algorithms that can be employed for scheduling and peer selection still awaits for a sound answer. How to provide differentiated service levels to different users is a further open question, and so appears the validation of peer-to-peer approaches for video applications with tight reliability constraints.

In this special issue we make an attempt to focus on a few specific issues of this realm: the contributions presented below shed some light on the examined problems, and let the reader appreciate the strength and novelty of their reach.

M. L. Merani $(\bowtie)$

Department of Information Engineering, University of Modena and Reggio Emilia,

Modena, Italy

e-mail: marialuisa.merani@unimore.it
The work "iGridMedia: the system to provide low delay peer-to-peer live streaming service over the Internet", by M. Zhang, L. Sun, S. Yang and Y. Fang, addresses the challenging issue of supporting interactive applications via a peer-to-peer streaming technology. An ad-hoc protocol, iGridMedia, is proposed and implemented, that satisfies delay and synchronization requirements more stringent than in current peer-to-peer solutions: its performance, in terms of consumed server bandwidth and required delay, is carefully investigated, outlining that even in the presence of peers with moderate upload capacity and peer churns the system behavior is excellent.

In "Adaptive Topology Formation for Peer-to-Peer Video Streaming", H. Luan, K.-W. Kwong, X. Hei and D. H. K. Tsang propose an adaptive peer-to-peer framework consisting of two major components: source rate variation and most importantly, adaptive overlay topology formation. At source level, the video server dynamically adjusts its emitting rate on the basis of local measurements of the peers' download rates. As regards topology, a link-level homogeneous overlay is built via a distributed algorithm, so that peers achieve an equal perconnection upload/download bandwidth and video flows experience no bottlenecks along the delivery path. Both analysis and simulations indicate that the proposed solution swiftly converges, maximizing the video playback quality.

The paper "Delay and playout probability trade-off in mesh-based peer-to-peer streaming with imperfect information", by I. Chatzidrossos, G. Dan and V. Fodor, details an analytical framework to evaluate the performance that different scheduling algorithms achieve in a mesh-based peer-to-peer streaming system. This work examines alternative solutions, where scheduling is performed at the 
transmitting peers, and for each strategy it determines how the playout probability is affected by the number of neighbours, the playback delay and the update frequency of buffer maps. The scalability of the examined algorithms is also investigated, and the model is employed to determine the effects that peer churns have on system performance.

In "A Decentralized Service Composition approach for future peer-to-peer video delivery", M. Kleis, B. Radier, S. Elmoumouhi, G. Carle and M. Salaun focus on QoS estimation techniques that decrease the time and measurement complexity of overlay-based service composition approaches. They put forth a distributed bandwidth estimation technique that combines landmark-based distance estimation and a projection technique; they further validate its accuracy via both theoretical analysis and simulation.

in "Analysis and Optimization of Peer-to-peer Streaming Efficiency Based on Buffer Model", H. Liu and G. Riley study the chance that peers have no pieces interesting for their neighbours and derive an upper bound for system efficiency modeling the buffers and exploiting probability theory.

Finally, in "Measurement and Enhancement of BitTorrent-based Video File Swarming", H.Wang, J. Liu and $\mathrm{K} . \mathrm{Xu}$ examine BitTorrent via a large scale measurement campaign that concentrates on video file swarms. The authors highlight the challenges in providing streaming services in addition to the current download-and-play mode, and suggest the key to overcome such hurdles, lying in a suitable peer organization method.

As a concluding remark, I hope this special issue spurs the curiosity of the reader as it did with the reviewers of its submitted papers: its lively and interesting contributions are worth being studied and discussed.
I am greatly indebted to the Editor-in-Chief Professor Sherman Shen for his experienced and firm guidance, as well for the careful attention he devoted to this issue. Also, a special thank has to be recognized to the editorial assistants Christina Chua and Melissa Fearon for their prompt and accurate help in all the phases of this publication.

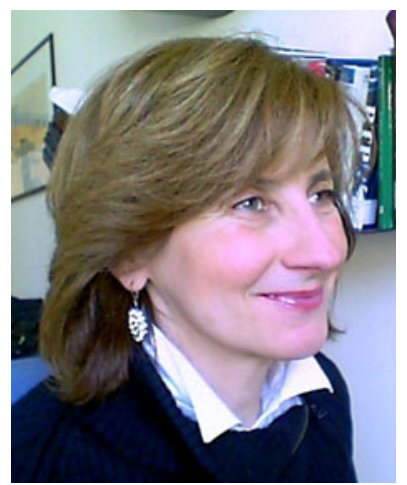

Maria Luisa Merani received both the M.Sc. (summa cum laude) and the Ph.D. in electrical engineering from the University of Bologna, Italy, in 1987 and 1992, respectively. In 1991 she spent one year at the Computer Science Department of the University of California in Los Angeles. Since 1993 she is with the Department of Information Engineering of the University of Modena and Reggio Emilia, where she is currently an associate professor. In the past, her research interests have spanned over the area of radio communications, with emphasis on wireless multimedia. At present she is involved in research on peer-to-peer systems for live video streaming. Dr. Merani has been very active within the Technical Program Committee of several flagship international conferences (IEEE Globecom, IEEE ICC, IEEE WCNC, IEEE ISSSTA, IEEE PIMRC). She served as the technical program co-chair for the 2nd IEEE International Symposium on Wireless Communication Systems 2005 (ISWCS'05) and for IEEE Globecom, 2007 and 2009 editions. In 2010 she has been the general chair of the fifth edition of the IEEE International Symposium on Wireless Pervasive Computing.

In October 2009 Cambridge University Press published her textbook "Hands on networking: from theory to practice". Dr. Merani is an IEEE Senior Member and an editor of the IEEE Transactions on Wireless Communications. 